Published in final edited form as:

Nat Rev Neurosci. 2006 January ; 7(1): 11-18. doi:10.1038/nrn1826.

\title{
Oligodendrocyte Wars
}

\author{
William D Richardson, Nicoletta Kessaris, and Nigel Pringle \\ Wolfson Institute for Biomedical Research and Department of Biology, University College London, \\ Gower Street, London WC1E 6BT, UK
}

\section{Summary}

Oligodendrocyte precursors first arise in a restricted ventral part of the embryonic spinal cord and migrate laterally and dorsally from there. Later, secondary sources develop in the dorsal cord. Normally the ventrally-derived precursors compete with and suppress their dorsal counterparts. There are ventral and dorsal sources in the forebrain too but here the more dorsal precursors prevail and the ventral-most lineage is eliminated during postnatal life. How do the different populations compete and what is outcome of the competition? Do different embryonic origins signify different functional subgroups of oligodendrocytes?

\section{Background}

The developmental origin of oligodendrocytes has been hotly debated for years. Some labs including our own favoured a unique origin of oligodendrocytes in the ventral neural tube, while others went for diversity and multiple origins. The published literature was conflicting and confusing. At last, new in vivo approaches are coming to the rescue. As is often the case, the answer turns out to be more complex than expected.

The established (pre-1990's) view was that oligodendrocytes were probably generated from all parts of the embryonic ventricular zones (VZ) (e.g. reference 1). This seemed inherently likely because mature oligodendrocytes are found in all regions of the adult central nervous system (CNS) with no obvious preference for position along dorsal-ventral or anteriorposterior axes. There were also some indications that radial glia, which are widespread throughout the developing CNS, can trans-differentiate into oligodendrocytes at the end of neurogenesis $2-4$. This, too, tended to favour widespread generation of oligodendrocytes.

This view was challenged in the early 1990's by the suggestion, amply confirmed since, that there is a specialized oligodendrogenic domain in the ventral VZ of the embryonic spinal cord 5-12 (for review see reference 13) and possibly also the forebrain 6,14-16. These ventral germinal zones produce migratory oligodendrocyte precursors that travel laterally and dorsally, sometimes over long distances, to populate all parts of the developing CNS before differentiating into myelin-forming oligodendrocytes. We ourselves favoured the view that ventrally-derived precursors produce most or all oligodendrocytes in the spinal cord and forebrain. This was a tidy idea because it had already been established that different parts of the VZ generate distinct types of neurons under the influence of different cocktails and concentrations of signaling molecules such as Sonic hedgehog (Shh) and Bone Morphogenetic Proteins (BMPs) 17. Since there was - and still is - no compelling evidence for functionally distinct classes of oligodendrocytes, this implied that they might have a 
singular origin. However, this has turned out to be an over-simplification, for several recent articles have now shown conclusively that there are dorsal as well as ventral origins in the spinal cord and brain.

This article aims to provide a historical perspective on the "origins" debate, which illustrates in microcosm the struggle for understanding that runs through all science. A series of recently published articles reveals that the jostling among rival ideas and labs is mirrored by another kind of competition among the oligodendrocyte populations themselves 18-21.

\section{Oligodendrocyte origins - a battle of ideas}

The idea of a single, ventral source for oligodendrocytes was controversial at the time and not everyone bought into it. In particular Zalc, Thomas and colleagues took a stand for diversity, arguing for multiple sources both dorsal and ventral 22,23. Some of their evidence rested on studies with a myelin proteolipid protein (PLP/DM 20) - lacZ reporter transgene; these studies could be questioned on the grounds that the transgene might not necessarily reflect the endogenous expression pattern of PLP/DM 20 and, even if it did, PLP/DM 20 is not necessarily restricted to oligodendrocyte lineage cells at all times and in all parts of the CNS. A major division of opinion developed in the field - single versus multiple origins of oligodendrocytes 23,24 . Fervent debate ensued ....

The controversy about oligodendrocyte origins was compounded by the finding that primary cultures of embryonic spinal cord cells contain a population of precursor cells that can generate oligodendrocytes and astrocytes but not neurons in culture 25,26 . These so-called glial restricted precursors (GRPs) can be found in cultures derived from different parts of the spinal cord neuroepithelium, both dorsal and ventral. This, on the face of it, appears to go against the idea of a restricted ventral origin for oligodendrocytes. Moreover, GRPs emphasize a developmental relationship between oligodendrocytes and astrocytes, whereas we and others had been emphasizing a relationship between oligodendrocytes and neurons (specifically motor neurons in the spinal cord). However, this dispute is probably more imaginary than real because it is possible that GRPs could be generated widely throughout the VZ yet constrained by the local environment to generate only (or mainly) oligodendrocytes in some parts (e.g. the ventral spinal cord) and mainly astrocytes in others 27. These spatial constraints would presumably be overruled in culture. In other words, the developmental potential of cells in vitro is often different than their actual fates in vivo.

Why should it matter where oligodendrocytes arise during development? Well, if they can be generated from different parts of the VZ under the influence of different signaling pathways, that might imply that completely different regulatory pathways can lead to the same cell type. That would indicate a surprising lack of specificity in the downstream readout of signal transduction pathways. Alternatively, oligodendrocytes with different developmental origins might have distinct functions or properties in vivo - an equally intriguing possibility. Either way, it is important to get the story straight.

The field has been in desperate need of a resolution. Thankfully, that resolution now seems to be on the way with a series of recently-published articles, reviewed below. 


\section{New approaches to the rescue}

Three recent papers - one from the laboratory of Mengsheng Qiu in Louiseville, Kentucky, one from Johan Ericson's lab in Stockholm and the other from our own lab -- provide persuasive new evidence that oligodendrocytes in the spinal cord are derived from both ventral and dorsal sources 18-20.

The former two articles describe mice that are doubly deficient for the homeodomain transcription factors Nkx6.1 and Nkx6.2 (Nkx6-null). The Nkx6 factors are normally expressed in the ventral part of the embryonic spinal cord VZ abutting the floor plate progenitor domains $\mathrm{p} 3$, pMN, p2 and p1 (Fig.1). $N k x 6$ transcription is activated by Shh signaling from the notochord and floor plate at the ventral midline. In turn, Nkx6 activates the basic helix-loop-helix (bHLH) transcription factor Olig2, which is absolutely required for the generation of both motor neurons and oligodendrocyte precursors from progenitors in the ventral progenitor domain pMN 31-33. Nkx6-null spinal cords therefore lose expression of Olig2 in pMN, so production of both motor neurons and oligodendrocytes from the ventral VZ is completely blocked 18,19 .

Surprisingly, oligodendrocyte precursors that express the usual markers PDGFRa and Olig2 continue to be produced in the dorsal spinal cord of $N k x 6$ null mice. The dorsal precursors co-express Pax7, confirming their dorsal origin. In wild type mice, some oligodendrocyte precursors in the dorsal part of the cord were also found to express Pax7, indicating that dorsal production is a normal phenomenon. These precursors were missed previously (e.g. reference 6), presumably because they are generated after their ventrally-derived counterparts and mingle with them unnoticed. There are fewer Pax7-expressing oligodendrocyte precursors in wild-type spinal cord than in $N k x 6$ mutant cord, suggesting that ventrally-produced oligodendrocyte precursors normally suppress their dorsal counterparts, perhaps because they compete more effectively for essential proliferation and/or survival signals such as PDGF 34,35. The ventral precursors start to be generated a couple of days earlier than the dorsal ones (E12.5 compared to $\sim$ E15) so they have plenty of time to get pre-established.

Additional evidence for dorsally-derived oligodendrocytes comes from Cre-lox fatemapping experiments in transgenic mice 20 . We generated mice that carry a transgene that expresses Cre recombinase under the control of regulatory elements surrounding the $D b x 1$ homeobox gene 20. In these mice Cre expression mirrors the normal pattern of $D b x$ expression, which is restricted to neuroepithelial precursors in p1, p0, dP6 and dP5 - i.e. four progenitor domains centred on the dorsal-ventral midline (Fig.1). Crossing the mice with a Cre-dependent reporter line (Rosa26-GFP or Rosa26-lacZ) permanently labels the $D b x$ precursor cells and all of their differentiated progeny. Unexpectedly, a small number of oligodendrocytes was labeled, as well as the expected radial glia, interneurons and astrocytes. The $D b x$-derived oligodendrocytes comprised around 3\% of all oligodendrocytes in the spinal cord and were spread less widely than the majority - they were mainly located in the lateral white matter radially opposite their site of origin in the VZ (Fig. 2). Some $D b x-$ derived, Olig2-positive cells retained a radial process and transiently co-expressed the radial 
glial cell marker RC2, indicating that they are formed by direct inter-conversion from radial glia - as suggested already many years ago 2,4 .

Not all parts of the dorsal VZ generate oligodendrocytes. The dorsally-derived precursors revealed in $N k x 6$-null mice seem to arise from progenitor domains dP3, dP4 and dP5 18. This suggests that the oligodendrocytes labeled in our $\mathrm{Dbx} 1$-Cre fate mapping experiments might be derived from $\mathrm{dP5}$, the only region of overlap. In more recent fate-mapping studies with Msx3-Cre transgenic mice it appears that 10-15\% of all oligodendrocytes in the cervical spinal cord originate in the dorsal half of the cord. Many of these are concentrated in the dorsal funiculus where they contribute up to $50 \%$ of the oligodendrocytes (Fogarty, M., PhD Thesis, University of London 2005).

So there are both ventral and dorsal origins of oligodendrocytes in the spinal cord and brainstem, as predicted by others 23,36 (Fig.2). Our own previous position, that "most or all" oligodendrocytes are generated in the ventral cord 24 must now be softened to "most but not all". This is a gratifying conclusion as everyone can claim credit for being at least partly correct.

\section{The role of $\mathrm{Nkx2.2}$}

There has also been controversy over oligodendrocyte origins at a more microscopic level -whether or not there is precise correspondence between the ventral oligodendrogenic domain and the ventral precursor domains $\mathrm{p} 3$ and/or $\mathrm{pMN}$. This question relates to the transcriptional regulation of gliogenesis itself, because different progenitor domains express and are defined by different sets of transcription factors - e.g. Nkx2.2 in p3, Nkx6.1 and Olig2 in pMN - and these factors are also involved in cell type specification and later differentiation events. Careful descriptive studies in mice mapped early-forming oligodendrocyte precursors (PDGFRa-positive) to the pMN domain, just dorsal to the Nkx2.2 - positive p3 domain 37. This led us to suggest that oligodendrocytes might have a special lineage relationship with somatic motor neurons. However, this was subsequently challenged by analogous studies in chick 38,39, showing that PDGFRa-positive precursors arise entirely within the $\mathrm{Nkx} 2.2$-expressing $\mathrm{p} 3$ domain in birds.

It turns out that the expression of $\mathrm{Nkx} 2.2$ changes with time, spreading dorsally to overlap with the pMN domain (defined by expression of Olig2) 33,40-42 during later embryogenesis. In mice, oligodendrocyte precursors in the cervical spinal cord are formed within pMN, after motor neuron production is over but starting before the dorsal expansion of Nkx2.2 41 (Fig.3). In chicks they are formed after expansion of Nkx2.2 and then only within the precise region of overlap with Olig2 39 (Fig.3) - neither p3 nor pMN but a new, hybrid p3/pMN domain. Here is a subtle species difference between rodents and birds. However, a common feature is that oligodendrocyte precursors develop from Olig2expressing neuroepithelium both in rodents and birds, so it seems likely that there is a close lineage connection between motor neurons and oligodendrocytes in both. Another common feature between chicks and mice is that Nkx2.2 is up-regulated in differentiating oligodendrocytes in the white matter 41. This fits with the notion that $\mathrm{Nkx} 2.2$ is important in maturation, not initial specification of the oligodendrocyte lineage in the mouse spinal cord 43. Vallstedt et al. 19 have now shown that the "chick pattern" of Nkx2.2 expression is 
preserved in the mouse brainstem, so that there is variation even along the mouse neuraxis. Whether this means that there are subtle differences in the properties of oligodendrocytes in the brainstem versus spinal cord is not known.

\section{Oligodendrocyte wars in the forebrain}

The controversy over oligodendrocyte origins extends to the forebrain. Here, too, there is evidence for a ventral source in the $\mathrm{VZ}$ of the basal forebrain. Cells that express oligodendrocyte lineage markers such as Olig1, Olig2, Sox10 and PDGFRa first appear in the neuroepithelium of the medial ganglionic eminence (MGE) appear to migrate laterally and dorsally from there into all parts of the developing forebrain, including the cerebral cortex, before birth 14. But is there also a dorsal source(s) in the forebrain?

In the chick, apparently not. Chick-quail grafting experiments indicate that all oligodendrocytes in the avian cortex are derived from precursors originating in the ventral telencephalon (anterior entopeduncular area, AEP) 16. However, Cre-lox fate mapping experiments using transgenic Emx1-Cre mice suggest that a significant fraction of oligodendrocytes in the corpus callosum and other cortical white matter tracts are derived from endogenous cortical precursors 44 . Other studies provide evidence for either a ventral or a dorsal source 14-16,36,45-48. Again, the lack of consensus is striking.

Recent fate mapping studies from our own laboratory help resolve the confusion 21 . We found, using an Nkx2.1-Cre transgenic mouse line that marks neural progenitors in the basal forebrain (including MGE, AEP and pre-optic area) that the first oligodendrocyte precursors to arrive in the cortex around E16 are indeed immigrants from ventral territories. These invaders populate the entire cortex by E18, but are then joined by a second wave of oligodendrocyte precursors from the lateral and/or caudal ganglionic eminence(s) (MGE/ CGE) (Gsh2-positive territory). At E18, therefore, all oligodendrocyte lineage cells in the cortex are ventral in origin. After E18, however, the contribution of ventral cells starts to decrease as they are joined by yet another wave of oligodendrocyte precursors that originates within the cortex itself (Emx1-positive neuroepithelium). So, once again, there are both ventral and dorsal sources - depending on when one looks (Fig.2).

Remarkably, we found that the original population of MGE/AEP-derived precursors disappears after birth, being rapidly eliminated from the cortex and more gradually from all other parts of the brain. Almost no trace can be found of the initial Nkx2.1-derived oligodendrocyte population anywhere in the adult 21 . This is reminiscent of the nervous system remodeling that occurs during the embryo-larva transition in Drosophila. Do the early-forming oligodendrocyte precursors in the mouse have some special function that is not required in the adult? Or is the MGE/AEP-derived population an evolutionary relic that lost its importance as new sources developed in the expanding brain? We revisit these questions later (see below, Evolution of oligodendrocyte development).

Running parallel to these embryonic studies, there has been a long-running and elegant series of experiments from Jim Goldman and others demonstrating that, in the postnatal forebrain, oligodendrocytes are generated from progenitor cells that reside near the tips of the lateral ventricles 50-54. What is the relationship between the embryonic and postnatal 
germinal zones? As neurogenesis comes to an end during late embryogenesis, the forebrain VZ regresses until only a remnant remains at the cortico-striatal boundary, remaining active and continuing to generate new oligodendrocytes (and other cell types) after birth and into adulthood. The postnatal VZ and its neighbouring subventricular zone (SVZ) is derived mainly from the embryonic LGE and lateral cortex, with no contribution from more ventral regions (references 21, 47 and unpublished). Thus, the most ventral, MGE/AEP-derived progenitors leave no descendants in the postnatal SVZ. This possibly contributes to the gradual loss of MGE/AEP-derived oligodendrocytes during postnatal life.

\section{Different sources -- different cells?}

In the spinal cord, expression of Olig2 in the ventral VZ depends on Shh signaling from the notochord and floor plate 10. The dorsally-derived oligodendrocyte precursors also express Olig2 (and other established lineage markers such as PDGFRa and Sox10) even though it seems unlikely that Shh can act at that distance from the floor plate. This suggests that oligodendrocyte lineage specification might be controlled by a different signaling system in the dorsal cord. A Hedgehog-independent pathway clearly does exist, because oligodendrocytes can be generated from dorsal spinal cord or telencephalic precursors cultured in the presence of cyclopamine, a drug that blocks all Hedgehog signaling by binding to its co-receptor Smoothened (Smo) 55,56. In addition, Cai et al.18 have shown that mouse ES cells derived from Smo null blastocysts can generate oligodendrocyte lineage cells in culture. It has been shown that Fibroblast Growth Factor (FGF) can induce oligodendrocyte precursors in culture independently of Shh (i.e. in the presence of cyclopamine) 55,56 so maybe FGF signaling is responsible for specifying oligodendrocytes in the dorsal spinal cord. BMP and Wnt signaling pathways might also be involved 19,57-60

Hedgehog signaling has also been shown to be required (or at least intimately involved) in oligodendrocyte specification in the ventral forebrain 14,15,61. Shh expression is not detected in the embryonic cerebral cortex so the late wave of cortical oligodendrogenesis might also be under different control - again, perhaps FGF. It is known, for example, that FGF can induce oligodendrocyte production in cultures of embryonic cortical cells 10,14 .

If the ventral and dorsal telencephalic lineages are specified differently, does this mean that they are intrinsically different cells - specialized oligodendrocyte subtypes with distinct molecular and/or functional properties? If they are, it seems that the differences are not critical because, when we killed either the ventral- or dorsal (cortex)-derived populations at source by targeted expression of a Diphtheria toxin transgene, neighbouring populations moved in to fill the space, a normal number and distribution of oligodendrocytes developed and the animals survived and behaved normally 21 .

\section{Evolution of oligodendrocyte development}

If, as discussed above, there is a dorsal source of oligodendrocytes in the mouse telencephalon but not in the chick - what might the significance of this species difference be? Mice (and mammals in general) have a greatly increased cortical volume compared to birds and this presumably calls for many more cells of all sorts, including oligodendrocytes, 
during cortical development. Migration distances would also have increased significantly within the larger cortex. These changes might have provided selective pressure for the evolution of an additional, local source of oligodendrocytes in the cortex, to supplement those that migrate in from the basal forebrain. There is a nice precedent for this. In rodents, all GABAergic cortical interneurons are thought to be immigrants from the basal forebrain 49. In humans, which have undergone an additional, huge cortical expansion compared to rodents, there is also local production of GABAergic interneurons within the neocortex 68 .

According to the above scheme, the ventral source of oligodendrocytes is "primitive" and the more dorsal sources were later evolutionary additions that were necessary to allow cortical expansion. By analogy, the primary source of oligodendrocytes in the spinal cord should be ventral (pMN) and the dorsal sources a later evolutionary addition. We have suggested before that the original selection for oligodendrocytes in the caudal neural tube might have been specifically to myelinate motor axons in order to facilitate rapid locomotion (escape) - hence their production side-by-side with motor neurons in pMN 24,69. This still seems an attractive speculation. But what was the evolutionary selection for an additional source in the dorsal spinal cord? Perhaps it is simply an inconsequential by-product of the "space race" going on in the brain. Or the dorsally-derived oligodendrocytes could have some specialized role that we don't know about yet.

An alternative scenario is that all neuroepithelial cells throughout the CNS, regardless of position, are programmed to generate relatively small numbers of oligodendrocytes and astrocytes after the end of neurogenesis. Thus, their default behaviour is to generate neurons followed by glial cells - this is the "classical" view of gliogenesis. The oligodendrocyte "factory" in pMN might then have been a later evolutionary response to pressure for more oligodendrocyte lineage cells, available earlier in development. According to this model we would expect to find small numbers of both astrocytes and oligodendrocytes being generated from all spinal cord neuroepithelial domains, with some domains - like pMN - specializing in production of extra oligodendrocytes or astrocytes.

The mechanism by which the embryonic, MGE-derived oligodendrocytes are eliminated from the brain after birth -- and the reason for their removal -- is a mystery. If the need for them has essentially been supplanted by more local sources, then they might have no special function in the cortex and might simply be out-competed for proliferation and survival signals by the local precursors. Competition between ventral and dorsal progenitors has already been noted in the spinal cord - except in that case the ventral progenitors seem to win out, at least in the short term (see above, New approaches to the "origins" debate). A more interesting (but less likely) idea is that the early-forming lineage has a specific function in the embryonic cortex that is no longer required postnatally -- akin to the nervous system re-modelling that occurs in invertebrates (e.g. Drosophila) as they metamorphose from embryo to larva to adult.

Another type of explanation for the elimination of MGE-derived oligodendrocytes is suggested by recent fate mapping experiments (reference 21 and unpublished data) that show that the postnatal SVZ is descended from cells in the embryonic LGE and cortex, with no contribution from the MGE. Therefore, if there were significant turnover of 
oligodendrocytes throughout the life of the animal, new SVZ-derived cells would be expected to gradually replace previous generations of oligodendrocytes and would lead to the gradual loss of MGE-derived cells over time. It should be stressed that we do not yet know whether there is any turnover of oligodendrocyte lineage cells in vivo; it is quite possible that oligodendrocytes survive for the lifetime of the axons that they ensheath. However, it is known that new oligodendrocytes continue to be generated throughout life5054 so it will be interesting to discover whether these are to replace lost oligodendrocytes or to supplement the existing population - to myelinate new axons, for example. Note that not all ventrally-derived cells are eliminated in the adult - MGE-derived cortical interneurons and basal forebrain neurons persist long-term, for example (reference 21).

\section{Conclusion}

The driving force for scientific progress is competition among ideas and individuals. That has certainly been true of our field of glial cell development. At last, the long-running arguments over the site(s) of origin of oligodendrocytes are being settled - the answer is that there are both dorsal and ventral sources that become active at different times during development and that compete with each other for territory ("oligodendrocyte wars").

The old arguments will soon be forgotten as the field moves on but they were important in passing, because controversy focuses the mind, attracts attention and brings newcomers into the field. Scientific progress is ultimately a group effort in which controversy and dispute play an essential part.

Perhaps we can draw a line under the "origins" debate and move on to new questions. How diverse are glia, especially astrocytes? Does developmental origin predict cell function? What are the molecular mechanisms of cell fate selection and neuron-glial fate switching? Do glial precursors in the adult CNS have a physiological or structural role in addition to generating new glia? Can they also generate neurons? Do adult precursor/stem cells "remember" their origins in the embryonic VZ and, if so, do they retain the lineage restrictions of their neuroepithelial ancestors? We look forward with anticipation to another decade of cut and thrust ...

\section{Acknowledgements}

We would like to thank our colleagues, past and present, for their individual scientific contributions and tremendous fun. We also thank our fellow-scientists across the world - some named in this article - for stimulation and collaboration. Work in the authors' lab has been supported by the UK Medical Research Council (MRC), the Wellcome Trust and the European Union. NK is supported by the Wellcome Trust Functional Genomics Initiative and NP by a programme grant from the MRC.

\section{About the authors}

William D (Bill) Richardson is Professor of Biology at University College London, where he holds a joint appointment as group leader in the Wolfson Institute for Biomedical Research and Head of the Department of Biology. For many years his research has focused on the cell- and developmental biology of CNS glia, particularly oligodendrocytes. His obtained his $\mathrm{PhD}$ in Biophysics from King's College London and was then a postdoc, first at the National 
Institutes of Health, USA, then at the National Institute for Medical Research, London. He joined UCL as a Lecturer in 1985.

Nicoletta Kessaris is a senior postdoc in the Richardson lab. Before that, Nicoletta was a PhD student and postdoc in the Department of Genetics, Cambridge University, where she worked on genes involved in kidney development (kid genes). It was there that she became skilled in mouse genetic manipulation. She is developing her own research interests in forebrain neurogenesis in preparation for starting her own lab.

Nigel Pringle is a senior postdoc in the Richardson lab. He obtained his $\mathrm{PhD}$ in the Dept of Zoology, UCL, in 1992. Nigel has been responsible for several of the early observations on oligodendrogenesis in the ventral spinal cord and is now working on astrocyte development and diversity.

\section{References}

1. Altman J. Proliferation and migration of undifferentiated precursor cells in the rat during postnatal gliogenesis. Exp Neurol. 1966; 16:263-278. [PubMed: 5928982]

2. Choi BH, Kim RC, Lapham LW. Do radial glia give rise to both astroglial and oligodendroglial cells? Dev Brain Res. 1983; 8:119-130.

3. Choi BH, Kim RC. Expression of glial fibrillary acidic protein by immature oligodendroglia and its implications. J Neuroimmunol. 1985; 8:215-235. [PubMed: 2409106]

4. Hirano M, Goldman JE. Gliogenesis in the rat spinal cord: Evidence for origin of astrocytes and oligodendrocytes from radial precursors. J Neurosci Res. 1988; 21:155-167. [PubMed: 3216418]

5. Warf BC, Fok-Seang J, Miller RH. Evidence for the ventral origin of oligodendrocyte precursors in the rat spinal cord. J Neurosci. 1991; 11:2477-2488. [PubMed: 1869925]

6. Pringle NP, Richardson WD. A singularity of PDGF alpha-receptor expression in the dorsoventral axis of the neural tube may define the origin of the oligodendrocyte lineage. Development. 1993; 117:525-533. [PubMed: 8330523]

7. Noll E, Miller RH. Oligodendrocyte precursors originate at the ventral ventricular zone dorsal to the ventral midline region in the embryonic rat spinal cord. Development. 1993; 118:563-573. [PubMed: 8223279]

8. Yu W-P, Collarini EJ, Pringle NP, Richardson WD. Embryonic expression of myelin genes: evidence for a focal source of oligodendrocyte precursors in the ventricular zone of the neural tube. Neuron. 1994; 12:1353-1362. [PubMed: 7516688]

9. Timsit $\mathrm{S}$, et al. Oligodendrocytes originate in a restricted zone of the embryonic ventral neural tube defined by DM-20 mRNA expression. J Neurosci. 1995; 15:1012-1024. [PubMed: 7869079]

10. Lu QR, et al. Sonic hedgehog-regulated oligodendrocyte lineage genes encoding bHLH proteins in the mammalian central nervous system. Neuron. 2000; 25:317-329. [PubMed: 10719888]

11. Takebayashi $\mathrm{H}$, et al. Dynamic expression of basic helix-loop-helix Olig family members: implication of Olig2 in neuron and oligodendrocyte differentiation and identification of a new member, Olig3. Mech Dev. 2000; 99:143-148. [PubMed: 11091082]

12. Zhou Q, Wang S, Anderson DJ. Identification of a novel family of oligodendrocyte lineage-specific basic helix-loop-helix transcription factors. Neuron. 2000; 25:331-343. [PubMed: 10719889]

13. Rowitch DH. Glial specification in the vertebrate neural tube. Nat Rev Neurosci. 2004; 5:409-419. [PubMed: 15100723]

14. Tekki-Kessaris N, et al. Hedgehog-dependent oligodendrocyte lineage specification in the telencephalon. Development. 2001; 128:2545-2554. [PubMed: 11493571]

15. Spassky N, et al. Sonic hedgehog-dependent emergence of oligodendrocytes in the telencephalon: evidence for a source of oligodendrocytes in the olfactory bulb that is independent of PDGFRalpha signaling. Development. 2001; 128:4993-5004. [PubMed: 11748136] 
16. Olivier C, et al. Monofocal origin of telencephalic oligodendrocytes in the chick embryo : the entopeduncular area. Development. 2001; 128:1757-1769. [PubMed: 11311157]

17. Jessell TM. Neuronal specification in the spinal cord; inductive signals and transcriptional codes. Nature Reviews Genetics. 2001; 1:20-29.

18. Cai J, et al. Generation of oligodendrocyte precursor cells from mouse dorsal spinal cord independent of Nkx6 regulation and Shh signaling. Neuron. 2005; 45:41-53. [PubMed: 15629701] [* This article describes studies with $N k x 6.1 / N k x 6.2$ compound knockout mice, demonstrating Sonic hedgehog-independent production of OLPs in the dorsal spinal cord.]

19. Vallstedt A, Klos JM, Ericson J. Multiple dorsoventral origins of oligodendrocyte generation in the spinal cord and hindbrain. Neuron. 2005; 45:55-67. [PubMed: 15629702] [* This article, like the previous one (Cai et al., 2005) describes studies with $N k x 6$ null mice that demonstrate production of oligodendrocyte precursors in the dorsal spinal cord and hindbrain and provides evidence for the involvement of BMPs in dorsal specification events. Vallstedt et al. also show that the role of the transcription factor Nkx2.2 differs between spinal cord and brainstem.]

20. Fogarty M, Richardson WD, Kessaris N. A subset of oligodendrocytes generated from radial glia in the dorsal spinal cord. Development. 2005; 132:1951-1959. [PubMed: 15790969] [* This article from our own lab provides independent evidence, by Cre-lox fate mapping in transgenic mice, for dorsal production of OLPs (and astrocytes). It also shows that specification of the dorsal subset of OLPs is Hedgehog-independent in culture but depends on FGF signalling.]

21. Kessaris N, et al. Competition among oligodendrocyte sub-populations in the forebrain and elimination of an early embryonic lineage. Nat Neurosci. 2006 [* This article describes experiments, using a series of Cre deleter mouse lines, to show that OLPs originate in both ventral and dorsal forebrain territories. Kessaris et al. also killed ventral and dorsal populations separately by targeted expression of Diphtheria toxin A chain, and showed that the different regional populations are able to substitute functionally for one another.]

22. Spassky N, et al. Multiple restricted origin of oligodendrocytes. J Neurosci. 1998; 18:8331-8343. [PubMed: 9763477]

23. Spassky N, et al. Single or multiple oligodendroglial lineages: a controversy. Glia. 2000; 29:143148. [PubMed: 10625332] [* This article and the next (Richardson et al. 2000) set out the contemporary arguments for and against multiple ventral and dorsal origins of oligodendrocytes versus a restricted ventral origin. These articles epitomise the "Wars" described in the current review.]

24. Richardson WD, et al. Oligodendrocyte lineage and the motor neuron connection. Glia. 2000; 12:136-142.

25. Rao MS, Noble M, Mayer-Proschel M. A tripotential glial precursor cell is present in the developing spinal cord. Proc Natl Acad Sci USA. 1998; 95:3996-4001. [PubMed: 9520481]

26. Liu Y, Rao M. Oligodendrocytes, GRPs and MNOPs. Trends Neurosci. 2003; 26:410-412. [PubMed: 12900171] [* Read this article for a discussion of the current debate about glial restricted precursors versus neuron-oligodendrocyte precursors. Whether these two concepts are incompatible or not depends on the precise meaning attached to lineage diagrams such as those presented in the present review article (Box 1).]

27. Rowitch DH, Lu QR, Kessaris N, Richardson WD. An 'oligarchy' rules neural development. Trends Neurosci. 2002; 25:417-422. [PubMed: 12127759]

28. Cameron-Curry P, Le Douarin NM. Oligodendrocyte precursors originate from both the dorsal and the ventral parts of the spinal cord. Neuron. 1995; 15:1299-1310. [PubMed: 8845154]

29. Pringle NP, Guthrie S, Lumsden A, Richardson WD. Dorsal spinal cord neuroepithelium generates astrocytes but not oligodendrocytes. Neuron. 1998; 20:883-893. [PubMed: 9620693]

30. Fu H, et al. Molecular mapping of the origin of postnatal spinal cord ependymal cells: evidence that adult ependymal cells are derived from Nkx6.1+ ventral neural progenitor cells. J Comp Neurol. 2003; 456:237-244. [PubMed: 12528188] [* This article provides persuasive evidence that the postnatal ependymal layer surrounding the lumen of the postnatal spinal cord is derived exclusively from neuroepithelial cells in the ventral (Nkx6.1-expressing) part of the embryonic spinal cord. The results of Cre-lox fate mapping (Fogarty et al., 2005; see above) support this conclusion, which raises interesting questions about the cell fate potential of neural stem cells in the adult.] 
31. Lu QR, et al. Common developmental requirement for oligodendrocyte lineage gene (Olig) function indicates a motor neuron/oligodendrocyte lineage connection. Cell. 2002; 109:75-86. [PubMed: 11955448]

32. Takebayashi H, et al. The basic helix-loop-helix factor Olig2 is essential for the development of motoneuron and oligodendrocyte lineages. Curr Biol. 2002; 12:1157-1163. [PubMed: 12121626]

33. Zhou Q, Anderson DJ. The bHLH transcription factors OLIG2 and OLIG1 couple neuronal and glial subtype specification. Cell. 2002; 109:61-73. [PubMed: 11955447]

34. Calver AR, et al. Oligodendrocyte population dynamics and the role of PDGF in vivo. Neuron. 1998; 20:869-882. [PubMed: 9620692]

35. van Heyningen P, Calver AR, Richardson WD. Control of progenitor cell number by mitogen supply and demand. Curr Biol. 2001; 11:232-241. [PubMed: 11250151]

36. Ivanova A, et al. Evidence for a second wave of oligodendrogenesis in the postnatal cerebral cortex of the mouse. J Neurosci Res. 2003; 73:581-592. [PubMed: 12929126]

37. Sun T, Pringle NP, Hardy AP, Richardson WD, Smith HK. Pax6 influences the time and site of origin of glial precursors in the ventral neural tube. Mol Cell Neurosci. 1998; 12:228-239. [PubMed: 9828088]

38. Xu X, et al. Selective expression of Nkx-2.2 transcription factor in chicken oligodendrocyte progenitors and implications for the embryonic origin of oligodendrocytes. Mol Cell Neurosci. 2000; 16:740-753. [PubMed: 11124894]

39. Soula C, et al. Distinct sites of origin of oligodendrocytes and somatic motor neurons in the chick spinal cord; oligodendrocytes arise from Nkx2.2-expressing progenitors by a Shh-dependent mechanism. Development. 2001; 128:1369-1379. [PubMed: 11262237]

40. Zhou Q, Choi G, Anderson D. The bHLH transcription factor Olig2 promotes oligodendrocyte differentiation in collaboration with Nkx2.2. Neuron. 2001; 31:791-807. [PubMed: 11567617] [* This pioneering article was the first to show a functional role for Nkx2.2 in oligodendrocyte development.]

41. Fu H, et al. Dual origin of spinal oligodendrocyte progenitors and evidence for the cooperative role of Olig2 and $N k \times 2.2$ in the control of oligodendrocyte differentiation. Development. 2002; 129:681-693. [PubMed: 11830569]

42. Agius E, et al. Converse control of oligodendrocyte and astrocyte lineage development by Sonic hedgehog in the chick spinal cord. Dev Biol. 2004; 270:308-321. [PubMed: 15183716]

43. Qi Y, et al. Control of oligodendrocyte differentiation by the Nkx2.2 homeodomain transcription factor. Development. 2001; 128:2723-2733. [PubMed: 11526078] [* This article provides evidence that $\mathrm{Nkx} 2.2$ plays an essential role in oligodendrocyte maturation, but not initial lineage specification in the mouse spinal cord.]

44. Gorski JA, et al. Cortical excitatory neurons and glia, but not GABAergic neurons, are produced in the Emx1-expressing lineage. J Neurosci. 2002; 22:6309-6314. [PubMed: 12151506]

45. He W, Ingraham C, Rising L, Goderie S, Temple S. Multipotent stem cells from the mouse basal forebrain contribute GABAergic neurons and oligodendrocytes to the cerebral cortex during embryogenesis. J Neurosci. 2001; 21:8854-8862. [PubMed: 11698597]

46. Wichterle H, Turnbull DH, Nery S, Fishell G, Alvarez-Buylla A. In utero fate mapping reveals distinct migratory pathways and fates of neurons born in the mammalian basal forebrain. Development. 2001; 128:3759-3771. [PubMed: 11585802]

47. Marshall CA, Goldman JE. Subpallial dlx2-expressing cells give rise to astrocytes and oligodendrocytes in the cerebral cortex and white matter. J Neurosci. 2002; 22:9821-9830. [PubMed: 12427838]

48. Yung SY, et al. Differential modulation of BMP signaling promotes the elaboration of cerebral cortical GABAergic neurons or oligodendrocytes from a common sonic hedgehog-responsive ventral forebrain progenitor species. Proc Natl Acad Sci U S A. 2002; 99:16273-16278. [PubMed: 12461181]

49. Marin O, Rubenstein JL. A long, remarkable journey: tangential migration in the telencephalon. Nat Rev Neurosci. 2001; 2:780-790. [PubMed: 11715055]

50. Levison SW, Goldman JE. Both oligodendrocytes and astrocytes develop from progenitors in the subventricular zone of postnatal rat forebrain. Neuron. 1993; 10:201-212. [PubMed: 8439409] 
51. Luskin MB, McDermott K. Divergent lineages for oligodendrocytes and astrocytes originating in the neonatal forebrain subventricular zone. Glia. 1994; 11:211-226. [PubMed: 7960027]

52. Levison SW, Goldman JE. Multipotential and lineage restricted precursors coexist in the mammalian perinatal subventricular zone. J Neurosci Res. 1997; 48:83-94. [PubMed: 9130137]

53. Parnavelas JG. Glial cell lineages in the rat cerebral cortex. Exp Neurol. 1999; 156:418-429. [PubMed: 10328946]

54. Levison SW, Young GM, Goldman JE. Cycling cells in the adult rat neocortex preferentially generate oligodendroglia. J Neurosci Res. 1999; 57:435-446. [PubMed: 10440893]

55. Chandran S, et al. FGF-dependent generation of oligodendrocytes by a hedgehog-independent pathway. Development. 2004; 130:6599-6609.

56. Kessaris N, Jamen F, Rubin L, Richardson WD. Cooperation between sonic hedgehog and fibroblast growth factor/MAPK signalling pathways in neocortical precursors. Development. 2004; 131:1289-1298. [PubMed: 14960493]

57. Gross RE, et al. Bone morphogenetic proteins promote astroglial lineage commitment by mammalian subventricular zone progenitor cells. Neuron. 1996; 17:595-606. [PubMed: 8893018]

58. Grinspan JB, et al. Stage-specific effects of bone morphogenetic proteins on the oligodendrocyte lineage. J Neurobiol. 2000; 43:1-17. [PubMed: 10756062]

59. Mekki-Dauriac S, Agius E, Kan P, Cochard P. Bone morphogenetic proteins negatively control oligodendrocyte precursor specification in the chick spinal cord. Development. 2002; 129:51175130. [PubMed: 12399304]

60. Shimizu T, et al. Wnt signaling controls the timing of oligodendrocyte development in the spinal cord. Dev Biol. 2005; 282:397-410. [PubMed: 15950605]

61. Nery S, Wichterle H, Fishell G. Sonic hedgehog contributes to oligodendrocyte specification in the mammalian forebrain. Development. 2001; 128:527-540. [PubMed: 11171336]

62. Bunge R. Glial cells and the central myelin sheath. Physiol Rev. 1968; 48:197-251. [PubMed: 4866614]

63. Bjartmar C, Hildebrand C, Loinder K. Morphological heterogeneity of rat oligodendrocytes: electron microscopic studies on serial sections. Glia. 1994; 11:235-244. [PubMed: 7960028]

64. Butt AM, Ibrahim M, Berry M. The relationship between developing oligodendrocyte units and maturing axons during myelinogenesis in the anterior medullary velum of neonatal rats. $\mathrm{J}$ Neurocytol. 1997; 27:327-338.

65. Butt AM, Colquhoun K, Tutton M, Berry M. Three-dimensional morphology of astrocytes and oligodendrocytes in the intact mouse optic nerve. J Neurocytol. 1994; 23:469-485. [PubMed: 7527074]

66. Kleopa KA, Orthmann JL, Enriquez A, Paul DL, Scherer SS. Unique distributions of the gap junction proteins connexin29, connexin32 and connexin47 in oligodendrocytes. Glia. 2004; 47:346-357. [PubMed: 15293232]

67. Fanarraga ML, Griffiths IR, Zhao M, Duncan ID. Oligodendrocytes are not inherently programmed to myelinate a specific size of axon. J Comp Neurol. 1998; 399:94-100. [PubMed: 9725703]

68. Letinic K, Zoncu R, Rakic P. Origin of GABAergic neurons in the human neocortex. Nature. 2002; 417:645-649. [PubMed: 12050665]

69. Richardson WD, Pringle NP, Yu W-P, Hall AC. Origins of spinal cord oligodendrocytes: possible developmental and evolutionary relationships with motor neurons. Dev Neurosci. 1997; 19:54-64.

70. Le Bras B, et al. Oligodendrocyte development in the embryonic brain: the contribution of the plp lineage. Int J Dev Biol. 2005; 49:209-220. [PubMed: 15906234] 


\section{Box 1}

\section{A Tale of Two Studies}

Nat Rev Neurosci. Author manuscript; available in PMC 2019 January 10. 


\section{Obliteration of the spinal cord lumen}

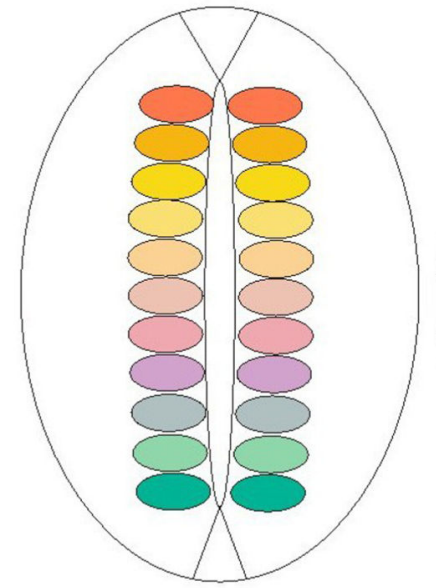

Embryo

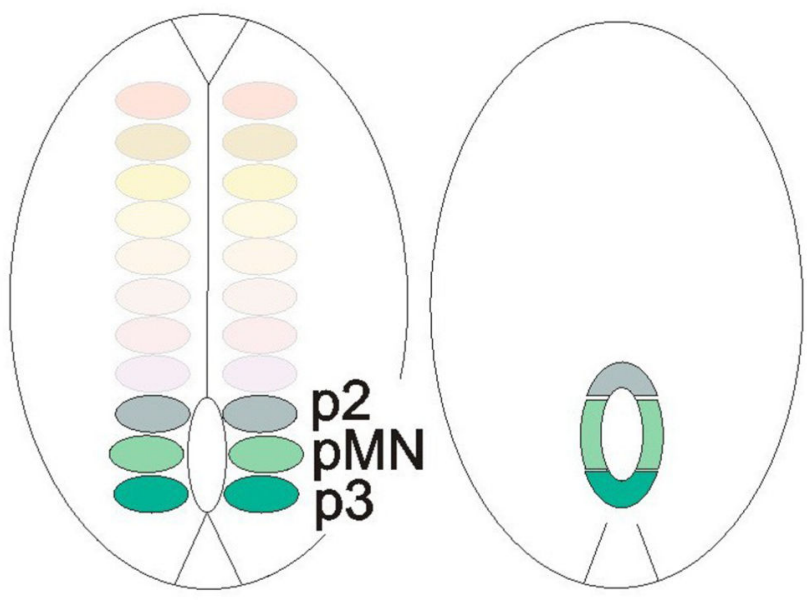

Adult

The two opposing views on oligodendrocyte origins were exemplified in two articles that appeared during the mid-1990's. Both described experiments designed to fate map the spinal cord neuroepithelium using chick-quail chimeras. The basic idea is simple: remove part of the ventral or dorsal spinal cord from a chick embryo in ovo, replace it with the equivalent part of a quail spinal cord and wait to see whether the oligodendrocytes that develop in the chimeric animal are of chick or quail origin. The first such study, by Cameron-Curry and Le Douarin 28, reported that oligodendrocytes are generated moreor-less equally from all parts of the dorsal and ventral VZ. The other study, from our own laboratory (Pringle et al. 29) claimed that oligodendrocytes are generated only from ventral VZ. How could such a stark discrepancy arise from what appear to be replicate sets of experiments?

One reason was that the criteria used to define a "dorsal" graft differed between the two studies - in an interesting way. Since one cannot observe the grafted neuroepithelial cells continuously from the time of surgery until the time of analysis (more than a week), some retrospective way of confirming the initial dorsal or ventral extent of the graft is required. Cameron-Curry and Le Douarin 28 presumed that the presence of graft-derived ependymal cells around the dorsal but not the ventral aspect of the spinal cord lumen at the time of analysis implied that the graft must have been dorsally restricted from the outset. However, this assumes that the dorsal ependymal layer is derived from dorsal neuroepithelial cells, an apparently reasonable assumption that nevertheless turns out to be wrong.

As the spinal cord matures, the central canal shrinks in size and the neuroepithelial cells that surround it are replaced by a layer of ependymal cells. It was recently shown that ependymal cells express the "ventral" transcription factors Nkx2.2, Olig2 and Nkx6.1 but not dorsal markers such as Pax7 30. Moreover, our recent fate mapping studies (see main text) demonstrate that progenitor domains more dorsal than p1 (i.e. beyond the dorsal 
expression limit of Nkx6.1) do not contribute to the postnatal ependymal layer (reference 17 and unpublished data). Taken together, the evidence suggests strongly that the ependymal layer is formed exclusively from ventral progenitors in $\mathrm{p} 3, \mathrm{pMN}$ and $\mathrm{p} 2$ (see Figure 1, Box 1).

Since Cameron Curry and Le Douarin's “dorsal” grafts gave rise to ependymal cells it follows that, far from being dorsally restricted, the grafts must in fact have spread deep into ventral territory. This could have resulted from preferential expansion of the grafted quail tissue in the chicken host, after transplant. The Pringle et al.29 study specifically excluded grafts that contributed to the ependymal layer.

Ironically, new genetic fate-mapping experiments - not subject to the uncertainties of microsurgery - now demonstrate that there are indeed some dorsally-derived oligodendrocytes in mice 18-20. Whether the Pringle et al.29 study simply overlooked this relatively small population or whether there really is a difference between rodents and birds remains to be seen (see main text, Oligodendrocyte wars in the forebrain and Evolution of oligodendrocyte development). 


\section{Box 2}

\section{Do all roads lead to Rome?}

Can cells that are born of progenitors in different parts of the embryo - under the influence of different positional signals and expressing different sets of patterning genes - ever converge on precisely the same phenotypic endpoint? Would we expect oligodendrocytes that are specified by Shh in the ventral neural tube to be identical to oligodendrocytes that are specified by different signals (e.g. FGF) in the dorsal neural tube? Different classes of neurons are derived from different parts of the neural tube so perhaps it would not be surprising if the glial products were different too. But what sorts of differences might we expect?

The morphology of oligodendrocytes varies according to the axons that they myelinate 62,63 . Those that ensheath large-diameter axons have a large cell body that lies close to the axon and they synthesize only a single internode's worth of myelin 63,64 . Other oligodendrocytes make many internodes - often more than thirty - on small-bore axons 65. There are also molecular differences between oligodendrocytes on large- versus small-bore axons - for example, in their gap junction proteins (connexins) 66. It is not known whether these are intrinsic differences or phenotypic variations of a single, plastic cell type. When oligodendrocyte precursors are purified from rodent optic nerve (which contains uniformly small-diameter axons) and transplanted into the ventral spinal cord (mixed large- and small-diameter axons), the grafted cells myelinate both large and small axons in the host 67 . This result smacks of phenotypic plasticity; however, it is also possible that the optic nerve contains a mixture of oligodendrocyte precursor subtypes but that the large-bore variety normally fail to find suitable axonal partners and lie dormant in the nerve. The general idea that there might be different subclasses of oligodendrocytes derived from different precursor subtypes (e.g. PDGF-dependent and -independent lineages 15) is an area of active debate 70.

Whether or not there are different subtypes of oligodendrocytes, it seems possible that there might be intrinsically different subtypes of astrocytes. A variety of tasks has been ascribed to astrocytes - inducing endothelial cells to form tight junctions and create the blood-brain barrier, buffering extracellular neurotransmitter concentrations, providing trophic support for neurons or oligodendrocytes. It remains to be seen whether these diverse functions are fulfilled by a single multi-tasking cell or else multiple cell types, perhaps derived from different neurogenic domains. 


\section{Online summary}

- $\quad$ Oligodendrocytes develop from precursor cells that migrate throughout the spinal cord and brain before differentiating and wrapping axons. The oligodendrocyte precursors (OLPs) themselves are generated from neuroepithelial cells that line the central canal of the spinal cord and the ventricles of the brain. Where exactly the OLPs originate in the ventricular zone (VZ) has been a contentious issue, which is now being resolved through the application of mouse genetics.

- The "classic" view was that gliogenesis was the default behaviour of all neuroepithelial cells after they had finished giving rise to neurons. Later, it was a specialized OLP "factory" was discovered in the ventral VZ of the spinal cord; this neuroepithelial domain (pMN) first generates motor neurons before switching to OLPs. Master regulatory genes (olig1, olig2) that control both motor neuron and OLP production are also expressed in the ventral forebrain, suggesting that ventral production of OLPs is the norm. However, a series of recent articles has debunked that idea by showing that they are generated widely through the VZ of the spinal cord and brain. In the spinal cord, most OLPs are produced in pMN but a minority ( 15\%) come from more dorsal parts of the VZ. In the forebrain, OLPs are generated from different parts of the embryonic VZ at different times, starting in the ventral forebrain and progressing dorsally into the cerebral cortex. Surprisingly, the earliest-formed OLPs and their progeny are eliminated during postnatal life.

- $\quad$ Specification of ventrally-derived OLPs requires Sonic hedgehog (Shh). However, OLPs from dorsal sources are hedgehog-independent, instead relying on FGF and other local signals. Whether this implies different functional subtypes of oligodendrocytes is not clear.

- In the forebrain, OLPs intermix and compete with one another for territory, because if OLPs from one part of the VZ are ablated in transgenic mice, the remaining OLPs quickly expand into the empty space and the mice survive and behave normally. This implies that there are no major functional differences among OLPs in the forebrain, even though they begin by expressing different region-specific transcription factors such as $n k x 2.1, g s h 2$ or emx 1 .

- $\quad$ There is some evidence that OLPs are generated only from ventral territories in birds. Why should they differ from mammals? We propose that ventral production was the "primitive" mode and that recruitment of more dorsal sources was required as the brain, particularly the cerebral cortex, increased in size during vertebrate evolution. 


\section{Spinal cord progenitor domains, transcription factors and cell fates}

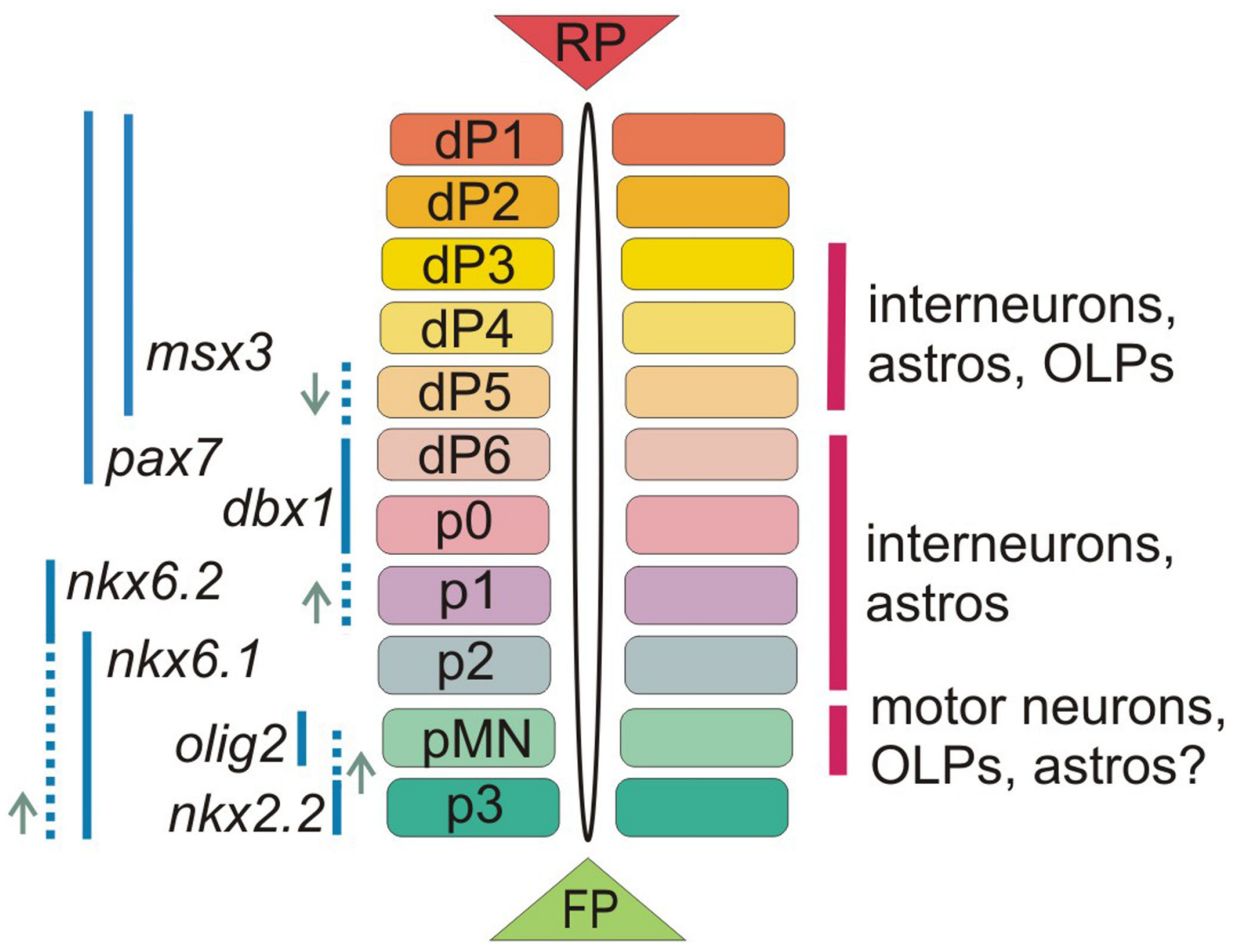

Figure 1.

Progenitor domains in the embryonic spinal cord and the cell types that they generate.

Neurons are formed before glia - astrocytes (astros) and oligodendrocyte precursors (OLPs). In general, OLPs are formed before astrocytes and ventral cell types before dorsal. Also shown are the expression domains of transcription factors mentioned in the text. Dotted lines indicate that the expression domain boundaries shift during development, in the direction of the small arrows - e.g. $N k x 2.2$ expression expands dorsally and $D b x 1$ expression contracts. Approximately $85 \%$ of all spinal cord oligodendrocytes are generated from pMN and the remainder from more dorsal progenitor domains. It is not known whether astrocytes are also generated from $\mathrm{pMN}$ but, if so, they are probably produced in small numbers relative to oligodendrocytes. FP, floor plate; RP, roof plate; dP1-dP6, dorsal progenitor domains; pMN and p0-p3, ventral progenitor domains. 

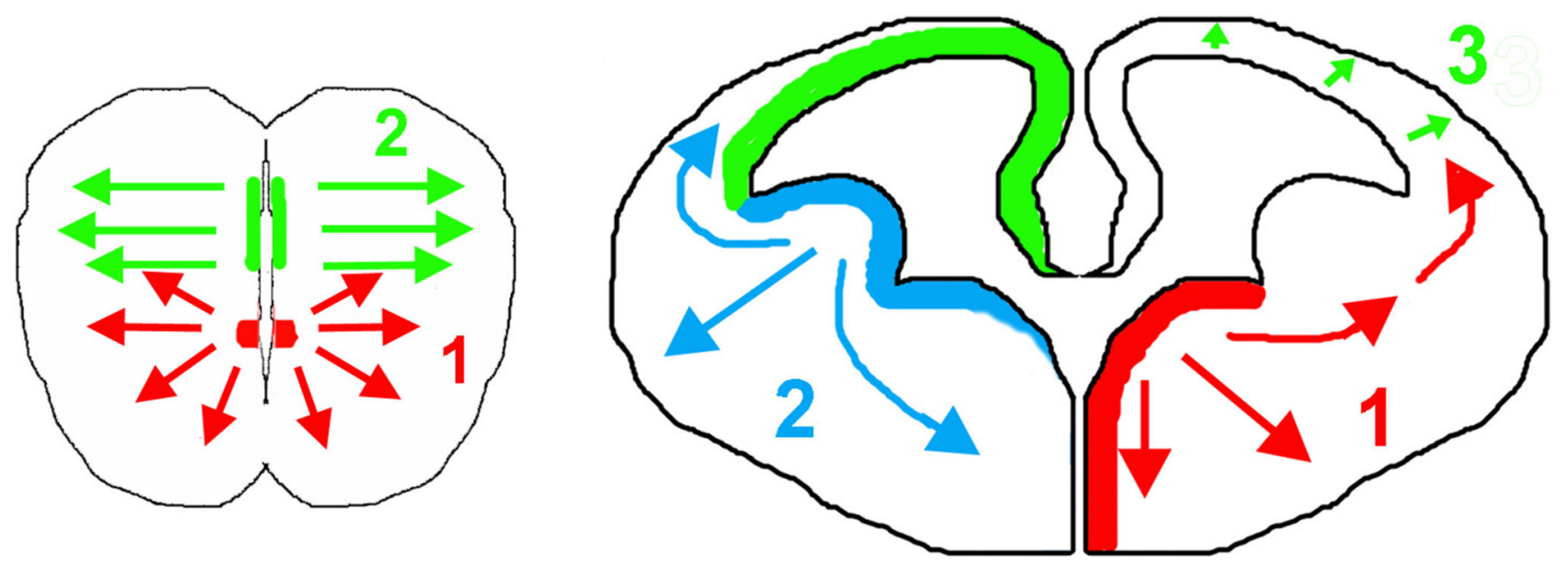

Figure 2.

Origins and migration of oligodendrocyte precursors in the rodent cervical spinal cord (left) and telencephalon (right). In the mouse spinal cord, approximately $85 \%$ of oligodendrocyte precursors are generated from pMN in the ventral VZ, starting around E12.5. Around E15, a secondary wave of precursors starts to be generated in more dorsal regions 16-18 by transdifferentiation of radial glia18. In the telencephalon, the ventral-most precursors in the MGE are produced from around E12.5, the LGE-derived precursors start to be produced a few days later and the cortex-derived precursors mainly after birth19. Diagram not to scale. 


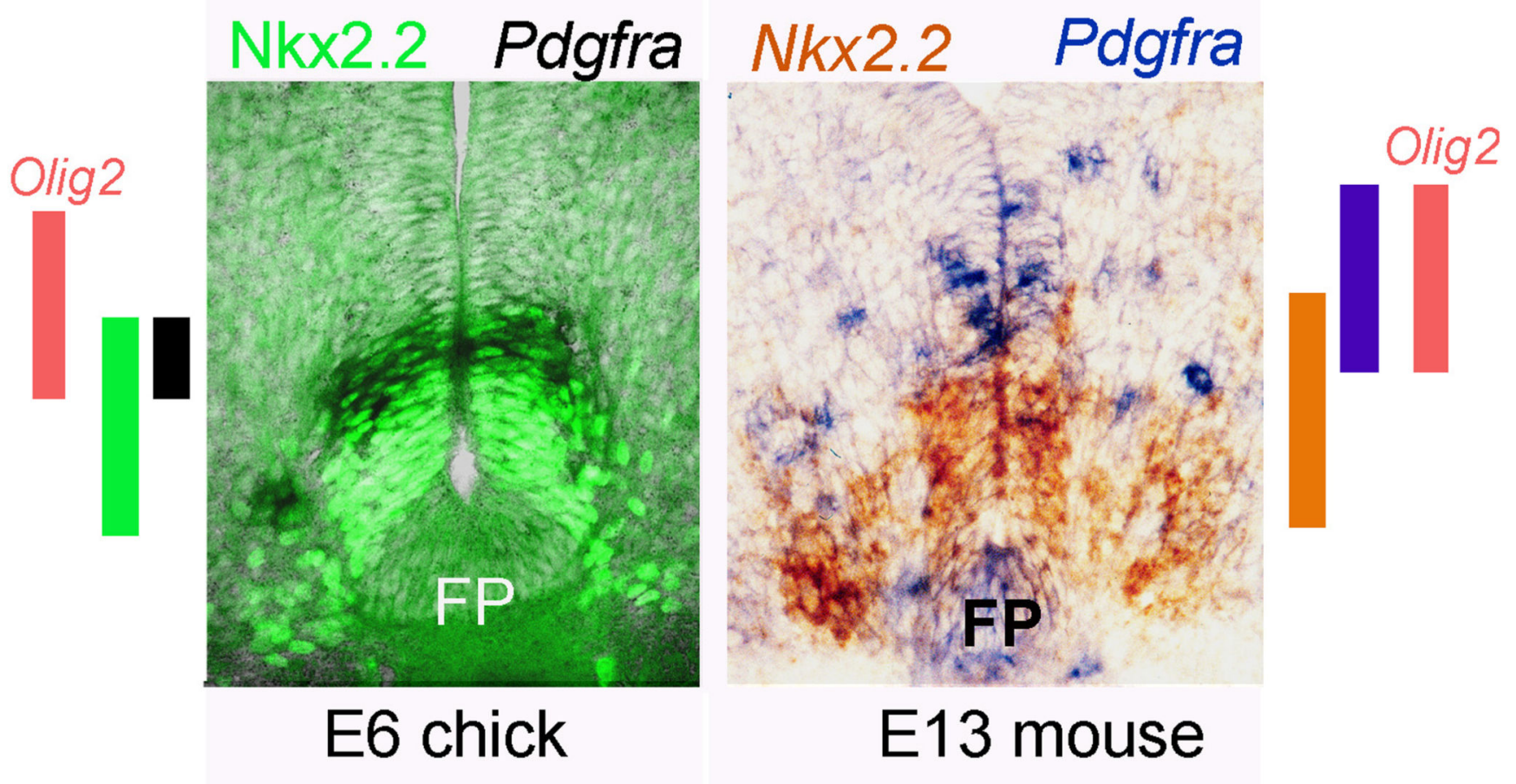

Figure 3.

Ventral origin of Pdgfra-positive oligodendrocyte precursors. In chick cervical spinal cord, Pdgfra+ precursors are derived exclusively from the dorsal part of the Nkx2.2-expressing domain, within the area of overlap between Nkx2.2 and Olig2 - a hybrid p3/pMN domain. In mouse cervical spinal cord, in contrast, they initially arise within the Olig2+ pMN domain, outside the dorsal limit of $\mathrm{Nkx} 2.2+$ expression (arrows). Later, after dorsal expansion of $\mathrm{Nkx} 2.2$ expression, they appear to arise within both the (Nkx2.2+, Olig2+) and (Nkx2.2--, Olig2+) domains. In both chicks and mice, oligodendrocyte precursors upregulate $\mathrm{Nkx} 2.2$ as they differentiate into myelinating oligodendrocytes in the white matter 30. Left panel: combined Nkx2.2 immunolabelling (green fluorescence) and Pdgfra in situ hybridization (black). Right panel: double in situ hybridization for Nkx2.2 (brown reaction product) and Pdgfra (blue). In the right panel, we assume that the blue Pdgfra+ cells in the floor plate region are oligodendrocyte precursors that have migrated ventrally from the ventral VZ, although it is also possible that they arose within the floor plate itself. 